\title{
Allele Sequencing of the Barley Stem Rust Resistance Gene Rpg1 Identifies Regions Relevant to Disease Resistance
}

\author{
A. Mirlohi, R. Brueggeman, T. Drader, J. Nirmala, B. J. Steffenson, and A. Kleinhofs
}

\begin{abstract}
First author: College of Agriculture, Isfahan University of Technology, Esfahan, Iran; second, third, and fourth authors: Department of Crop and Soil Sciences, Washington State University, Pullman; fifth author: Department of Plant Pathology, University of Minnesota, St. Paul; and sixth author: School of Molecular Biosciences and Department of Crop and Soil Sciences, Washington State University, Pullman. The first two authors contributed equally to this work.
\end{abstract}

Accepted for publication 2 April 2008.

\section{ABSTRACT}

Mirlohi, A., Brueggeman, R., Drader, T., Nirmala, J., Steffenson, B. J., and Kleinhofs, A. 2008. Allele sequencing of the barley stem rust resistance gene Rpgl identifies regions relevant to disease resistance. Phytopathology 98:910-918.

The stem rust resistance gene Rpgl has protected North American barley cultivars from significant yield losses for over 65 years. The remarkable durability of this gene warrants further study as to its possible origin and allelic variation. Eight Swiss barley (Hordeum vulgare) landraces and eight wild barley (H. vulgare subsp. spontaneum) accessions from diverse geographic regions were analyzed to uncover new alleles of Rpg 1 and learn about its possible origin. The two germplasm groups included accessions that were resistant and susceptible to Puccinia graminis f. sp. tritici pathotype MCCF. Allele-specific primers were utilized to amplify 1 kbp overlapping fragments spanning the Rpgl gene and sequenced if a polymerase chain reaction (PCR) fragment was generated. Variation among the PCR products revealed significant polymorphisms among these Hordeum accessions. Landraces and wild barley accessions sus- ceptible to pathotype MCCF exhibited the highest degree of Rpg1 polymorphism. One resistant landrace (Hv672) and one resistant wild barley accession (WBDC040) yielded all seven Rpgl-specific PCR fragments, but only landrace Hv672 coded for an apparently functional Rpg1 as determined by comparison to previously characterized resistant and susceptible alleles and also resistance to HKHJ, a stem rust pathotype that can specifically detect Rpgl in the presence of other resistance genes. Accessions resistant to stem rust pathotype MCCF, but completely lacking Rpgl-specific PCR amplification and hybridization with an Rpglspecific probe, suggested the presence of stem rust resistant gene(s) different from Rpgl in the Hordeum germplasm pool. Some Rpgl alleles that retained the ability to autophosphorylate did not confer resistance to Puccinia graminis $\mathrm{f}$. sp. tritici pathotype MCCF, confirming our previous observations that autophosphorylation is essential, but not sufficient for disease resistance. Thus, the RPG1 protein plays a complex role in the stem rust disease resistance-signaling pathway.

Additional keywords: functional analysis, $\mathrm{R}$ genes.
Stem rust (caused by the wheat stem rust fungus Puccinia graminis f. sp. tritici) has been one of the most important pathogens of barley (Hordeum vulgare L.) in the Upper Midwest region of the United States and Prairie Provinces of Canada over the past century. A single dominant gene (Rpgl) introgressed into barley cultivars has effectively controlled the stem rust epidemics which devastated the crop in the Northern Great Plains prior to 1942 (18). Many qualitative rust resistance genes in other cereal crops have been overcome by virulent pathotypes in a relatively short period of time (12); however, Rpgl has been durable, protecting barley from serious stem rust losses for more than 65 years. This remarkable durability warrants further characterization of Rpgl.

Rpgl was mapped to the short arm of barley chromosome $1(7 \mathrm{H})$ and cloned from cultivar Morex by construction of high-resolution genetic and physical maps (2). Highly resistant transgenic plants were obtained by transforming the susceptible barley cultivar Golden Promise, devoid of Rpgl, with the Morex Rpgl gene, demonstrating that the gene isolated was functional in conferring stem rust resistance (9). Rpg1 contains 14 exons in 4,466 bp of genomic sequence encoding an 837 amino acid protein with two tandem kinase domains, which is a unique resistance gene structure (2).

Sequence analysis of Rpgl from resistant North American barley cultivars revealed no amino acid sequence polymorphism (2). This suggests a singularity of the Rpgl source that can be

Corresponding author: B. Steffenson; E-mail address: bsteffen@umn.edu

doi:10.1094/PHYTO-98-8-0910

(C) 2008 The American Phytopathological Society traced to an unimproved bulked seed lot (from which the sister selections of Chevron [PI 38061] and Peatland [PI 539109] were derived) obtained by the United States Department of Agriculture from Switzerland in 1914. Another potential source of stem rust resistance in barley was the cultivar Kindred (CIho 6969), a farmer selection of a single stem rust resistant plant from a field of susceptible Wisconsin 37 barley (18). The plant from which Kindred was derived was probably an admixture because it differed from Wisconsin 37 for many more traits than just stem rust resistance. Moreover, sequence analysis revealed that the Rpgl in silico translated amino acid sequence in Kindred was identical to that of Chevron and Peatland (2). The source of Rpgl in Kindred was likely Chevron because this cultivar had been used in the Wisconsin barley breeding program for a number of years prior to the release of Wisconsin 37.

During the map-based cloning effort of Rpgl, allele sequencing revealed three groups of stem rust susceptible barley lines (2). The first group contained susceptible rpgl genes with 3 base pairs (GTT) inserted in the sixth exon at nucleotide position 1,546 relative to the Rpgl sequence of Morex. The second group contained an Rpg 1-like gene with a highly diverged 5' region (2), and the third group had no detectable Rpgl gene sequences. The second group, including barley line $\mathrm{Sm} 89010$, was recently characterized as containing a recombinant Rpgl gene with ABC1037, an $R p g 1$ gene family member located $\approx 50 \mathrm{kbp}$ proximal to $R p g 1$ (1). Lines with this recombinant Rpgl gene have a $5^{\prime}$ end highly homologous to $\mathrm{ABC} 1037$ and a $3^{\prime}$ end with high homology to Rpgl (1). Reverse transcription (RT)-PCR and sequence analysis indicated that the Sm89010 recombinant Rpgl gene is tran- 
scribed, but does not function in conferring stem rust resistance (to pathotype MCCF) because it and two other barley cultivars (Dicktoo and Gobernadora) containing the Sm89010-like Rpg1 gene were susceptible (2). It is possible that the Sm89010 Rpg1 gene expresses novel specificities to other stem rust pathotypes, as unequal exchange events have been suggested as a means for the evolution of plant disease resistance genes $(7,15)$. In flax, 13 alleles at the $L$ rust resistance locus have been reported to express 10 different rust resistance specificities. These resistance specificities are presumed to have evolved through various mechanisms, including single base substitutions, insertion/deletions (indels), unequal recombination, and reassortment of variable regions within the alleles by multiple intra-genic sequence exchange events (7).

Naturally occurring allelic diversity in plants has been suggested to be an important genetic component for phenotypic variation and crop improvement. It is therefore important to characterize this diversity in order to identify economically important alleles and polymorphisms (3). In addition to landraces of domesticated barley ( $H$. vulgare), the wild progenitor $H$. vulgare subsp. spontaneum is known to be a valuable source for alternative forms of disease resistance genes for barley improvement $(8,22)$. Stem rust resistance has been reported in wild barley, but the frequency of resistance is often low $(<10 \%)(8,20)$. Given the recent threat of new pathotypes such as $P$. graminis f. sp. tritici TTKS (i.e., isolate Ug99) in the world, wild barley may become an important new source of stem rust resistance genes (20).

The lack of Rpgl polymorphism among previously-tested stem rust resistant barley cultivars suggests either a highly conserved or single source for this gene. High conservation of a protein kinase disease resistance gene has been shown with the tomato Pto gene, which confers resistance to Pseudomonas syringae pv. tomato. The wild tomato species Lycopersicon pimpinellifolium is the source of the Pto gene in cultivated tomato (L. esculentum). A Pto ortholog LhirPto, isolated from another wild tomato relative (L. hirsutum), shares $97 \%$ amino acid identity with the Pto from L. pimpinellifolium (16). Even more surprising, the domesticated tomato has a highly conserved, transcriptionally active pto allele that fails to confer disease resistance (10). Since the Lycopersicon species separated presumably millions of years ago, these findings suggest that the PTO kinase has been highly conserved, even when it does not retain disease resistance function.

The objectives of this study were to use molecular techniques to uncover new alleles of Rpgl and learn about its possible origin in eight Swiss barley (H. vulgare) landraces and eight wild barley (H. vulgare subsp. spontaneum) accessions from diverse geographic regions. The presence/absence of Rpgl, its primary structure, and expression were analyzed by PCR of genomic DNA and mRNA (RT-PCR), Southern blotting, sequencing, western blotting, and autophosphorylation analyses.

\section{MATERIALS AND METHODS}

Plant materials and disease phenotyping. From a germplasm collection of 74 barley landraces from Switzerland and 318 ecogeographically diverse wild barley accessions (20), 16 (eight from each group) were selected and used in this study (Table 1). Landraces from Switzerland were chosen for analysis because they are the donors of Rpgl (i.e., Chevron and Peatland) in North American barley cultivars and may possess additional allelic diversity for the gene. Wild barley accessions also were included because of their great allelic diversity and potential for identifying the origin of Rpgl in cultivated barley. Within each germplasm group, four resistant and four susceptible accessions to stem rust pathotype MCCF were chosen from ecogeographically diverse locations to identify functional Rpgl variants as well as spontaneous mutations that result in loss of function. Pathotype MCCF has been used for a number of years to select breeding lines with Rpg1, develop high resolution maps for map-based cloning of the resistance gene, and conduct functional analyses with the cloned gene $(2,13,19)$. Recently, a comprehensive evaluation was made on the reaction of known stem rust resistance genes in barley to diverse pathotypes of $P$. graminis f. sp. tritici and isolates of $P$. graminis f. sp. secalis (the rye stem rust fungus) (21). This study revealed that $P$. graminis $\mathrm{f}$. sp. tritici pathotype HKHJ was more effective than MCCF for detecting Rpgl in the presence of several other known stem rust resistance genes (21). Thus, the germplasm initially selected for this study based on reaction to pathotype MCCF was subsequently evaluated to pathotype HKHJ to further resolve the functionality of Rpg1. Cultivars Morex (with Rpg1) and Steptoe (without any known resistance genes) also were included as the resistant and susceptible controls, respectively. Plants were grown and assayed for their reaction to stem rust as previously described (19).

DNA and RNA were isolated from a single plant of each of the 16 selected accessions based initially on their stem rust phenotype. The plants were grown in plastic pots containing potting mix in a growth chamber under cool fluorescent lights with a $16 \mathrm{~h}$

TABLE 1. Reaction of Swiss landrace and wild barley accessions to Puccinia graminis f. sp. tritici pathotypes MCCF and HKHJ at the seedling stage

\begin{tabular}{|c|c|c|c|c|c|}
\hline \multirow[b]{2}{*}{ Line } & \multirow[b]{2}{*}{ Origin } & \multicolumn{2}{|r|}{ Pathotype MCCF } & \multicolumn{2}{|c|}{ Pathotype HKHJ } \\
\hline & & IT range $^{a}$ & General reaction & IT range $^{a}$ & General reaction \\
\hline Swiss Hv584 & Fuormis, Switzerland & $0 ; 1$ to 10 & Resistant & $3^{-}$to 3 & Susceptible \\
\hline Swiss Hv587 & Platta, Switzerland & $0 ; 1$ to 21 & Resistant to moderately resistant & $3^{-}$to 3 & Susceptible \\
\hline Swiss Hv619 & Fetan, Switzerland & 10 & Resistant & $3^{-}$to $33^{+}$ & Susceptible \\
\hline Swiss Hv672 & Surava, Switzerland & $10 ;$ to 12 & Resistant & $10 ;$ to 21 & Resistant to moderately resistant \\
\hline Swiss Hv489 & Trans, Switzerland & 3 & Susceptible & 3 to $33^{+}$ & Susceptible \\
\hline Swiss Hv492 & Scheid, Switzerland & 3 to $33^{+}$ & Susceptible & $3^{-}$to 3 & Susceptible \\
\hline Swiss Hv611 & Plan, Switzerland & $3^{-}$to 3 & Susceptible & $3^{-}$to 3 & Susceptible \\
\hline Swiss Hv645 & Scuol, Switzerland & $3^{-}$to 3 & Susceptible & $3^{-}$to 3 & Susceptible \\
\hline WBDC040 & Haifa, Israel & $0 ; 1$ to 21 & Resistant to moderately resistant & $3^{-}$to 3 & Susceptible \\
\hline WBDC214 & Samarkand, Uzbekistan & $0 ; 1$ to $213^{-}$ & Resistant to moderately resistant & 3 & Susceptible \\
\hline WBDC220 & Chimkent, Kazakhstan & $10 ; 2$ to $213^{-}$ & Resistant to moderately resistant & 3 to $33^{+}$ & Susceptible \\
\hline WBDC225 & Dushanbe, Tajikistan & $0 ; 1$ to 21 & Resistant to moderately resistant & 3 to $3^{+}$ & Susceptible \\
\hline WBDC019 & West Azerbaijan, Iran & 3 to $33^{+}$ & Susceptible & $33^{+}$ & Susceptible \\
\hline WBDC 160 & Sweida, Syria & 3 to $3^{+} 3$ & Susceptible & 3 to $3^{+}$ & Susceptible \\
\hline WBDC269 & Lebanon & $3-2$ to $33^{+}$ & Moderately susceptible to susceptible & $3^{-}$to 3 & Susceptible \\
\hline WBDC323 & Ahalskii, Turkmenistan & 3 to $33^{+}$ & Susceptible & 3 to $3^{+}$ & Susceptible \\
\hline Morex $^{b}$ & Minnesota, USA & $0 ; 1$ to $213^{-}$ & Resistant to moderately resistant & 12 to 21 & Resistant to moderately resistant \\
\hline Steptoe ${ }^{b}$ & Washington, USA & 3 to $33^{+}$ & Susceptible & 3 to $33^{+}$ & Susceptible \\
\hline
\end{tabular}

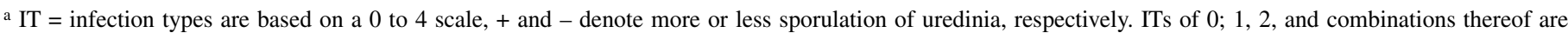
indicative of resistance, whereas ITs of 3, 4 and combinations thereof are indicative of susceptibility (21).

b Morex (with Rpg1) and Steptoe (without any known stem rust resistance gene) are the resistant and susceptible controls, respectively. 
photoperiod and day/night temperature of $21 \pm 1^{\circ} \mathrm{C} / 18 \pm 1^{\circ} \mathrm{C}$. For protein analysis by western blot and autophosphorylation, a single plant from the same seed source was grown under the above conditions.

DNA and RNA isolation. Genomic DNA for Southern hybridization was isolated from young barley leaves with a modified CTAB method (11). For PCR amplification, genomic DNA was isolated from young barley leaves according to Edwards et al. (6). Total RNA was isolated from young seedling leaves using Trizol reagent (4). Integrity and quantity of the RNA samples were checked by $1 \times$ TBE $(0.089$ M Tris-borate, $0.089 \mathrm{M}$ boric acid, and 0.002 M EDTA) agarose gel electrophoresis.

PCR and RT-PCR. PCR primers were designed from cultivar Morex and line Sm89010 Rpg1 allele sequences (GenBank accession numbers AF509748 and AF509762, respectively) to amplify approximately 1-kbp overlapping fragments covering the entire Rpg1 gene (Fig. 1 and Table 2). Primer set 228F6/228R6 amplified a fragment from both cultivars Morex and Sm89010; however, the Sm89010 fragment is of higher molecular weight (Table 2). Primer combination 37F2/228R6 amplified only the Sm89010 Rpgl allele and resulted in a 1,678-bp band. DGK-F1 and DGSex 2 primers are internal to $37 \mathrm{~F} 2 / 228 \mathrm{R} 6$ and were used only in the sequencing reaction in order to cover the gap between the forward 37F2 and reverse 228R6 primers. PCR reactions and analyses were as previously described (2).

Approximately $1 \mu \mathrm{g}$ of total RNA was used for oligo dT-primed first-strand cDNA synthesis using the Reverse Transcription System (Promega, Madison, WI) according to the manufacturer's recommended conditions followed by PCR reactions (2). Primers EX3cw2/228R6 and 37-F2/228R7 were used for RT-PCR to amplify Morex or Sm89010 Rpgl-specific cDNA, respectively.
Southern blot analysis. Southern blotting and hybridization were as previously described (11). Five micrograms of HindIIIdigested barley genomic DNA was separated by agarose gel electrophoresis, blotted onto nylon membranes, and hybridized with the Rpg1-specific DNA probe RSB228 (2).

Sequencing. PCR fragments were eluted from the agarose gel, purified, concentrated by ethanol precipitation, and used as templates for sequencing reactions. Sequencing reactions were performed using the Big-Dye Terminator kit (Applied Biosystems, Foster City, CA) and sequenced at the Bioanalytical Center, Washington State University, Pullman, WA. Sequences were analyzed and contigs assembled using ContigExpress from the Vector NTI software package.

cDNA cloning and sequencing. Oligo dT-derived cDNA from Morex and WBDC040 was used as the template to amplify the ninth intron/exon junction of Rpg1. RT-PCR products were cloned in the pGEM-T vector (Promega) following the manufacturer's protocol. Clones were confirmed by restriction digestion with EagI (New England Biolabs, Beverly, MA) and NcoI (Fermentas, Hanover, MD) and sequenced using T7 and SP6 universal primers.

Protein analysis. Polyclonal antibody was raised in New Zealand white rabbits at the Washington State University animal facility against a recombinant His-RPG1 protein purified from Pichia pastoris (13). Ribi adjuvant was used according to the manufacturer's recommendation (Corixa, Hamilton, MO). Western blotting and autophosphorylation assays were performed as previously described (13).

Rapid amplification of cDNA $3^{\prime}$ ends (3' RACE). 3' RACE was carried out using the FirstChoice RLM-RACE Kit (Ambion, Austin, TX) and the $3^{\prime}$ RACE System (Invitrogen, Frederick,

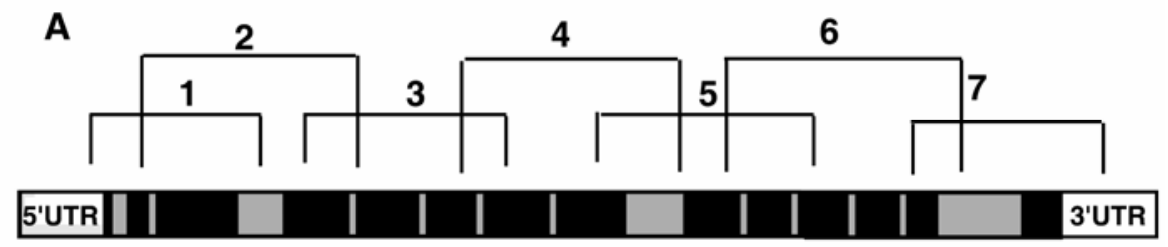

B

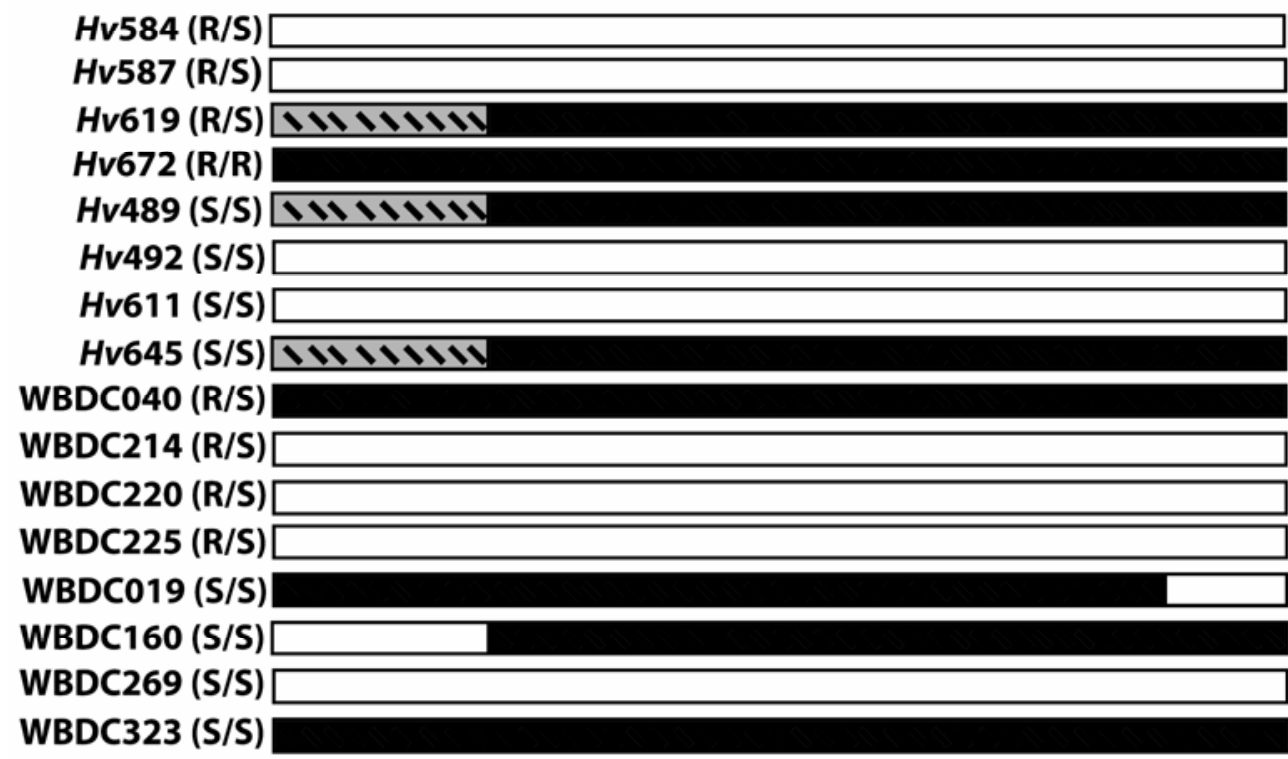

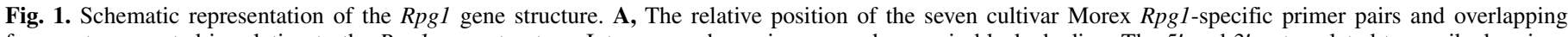

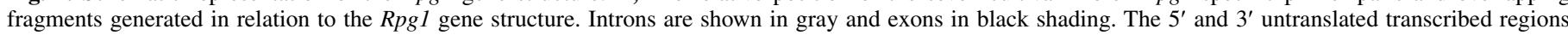

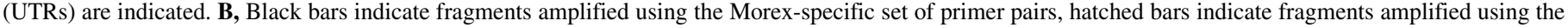

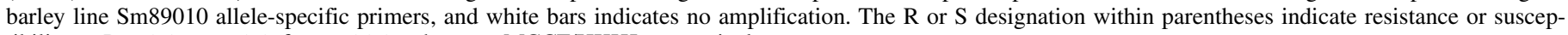
tibility to Puccinia graminis f. sp. tritici pathotypes $\mathrm{MCCF} / \mathrm{HKHJ}$, respectively. 
MD) under the manufacturer's recommended standard conditions. The nested gene-specific primers used for both systems were 228F3 and 228-F4 as previously described in Brueggeman et al. (2).

\section{RESULTS}

Stem rust reaction. The controls reacted as expected with Morex exhibiting resistant to moderately resistant infection types and Steptoe susceptible infection types to pathotypes MCCF and HKHJ (Table 1). Hv584, Hv587, Hv619, and Hv672 were among the most resistant accessions found to $P$. graminis $\mathrm{f}$. sp. tritici pathotype MCCF in the Swiss landrace germplasm, giving infection types ranging from $0 ; 1$ to 21 (Table 1). In contrast, Hv489, Hv492, Hv611, and Hv645 were among the most susceptible accessions found, giving infection types of 3- to 33+. Resistance to wheat stem rust in wild barley is fairly rare $(8,20)$. The resistant wild barley accessions used in this study (WBDC040, WBDC214, WBDC220, and WBDC225) were selected from a previous preliminary study examining a large wild barley germplasm collection (20) and were among the most resistant found to pathotype MCCF, giving infection types ranging from 0;1 to 213-. Accessions WBDC019, WBDC160, WBDC269, and WBDC323 were representative of the most susceptible accessions found and ex- hibited infection types ranging from $3-2$ to $3+3$. All Swiss landraces and wild barley accessions also were evaluated to HKHJ, a pathotype that can effectively detect Rpgl in the presence of other stem rust resistance genes (21). The Swiss landrace Hv672 was the only accession found with resistance (IT range of 10; to 21) to pathotype HKHJ. This suggests that it may be the only accession with a functional Rpgl gene (Table 1).

Lines with an apparently intact Rpg1 gene. Seven primer pairs, designed to amplify the entire Rpgl coding region, were used to amplify Rpgl genes from all accessions. All expected DNA fragments were successfully amplified from Swiss Hv672, WBDC040, and WBDC323 (Fig. 1B), suggesting the presence of an intact Rpgl gene in these genotypes. Southern blots of genomic DNA digested with HindIII and hybridized with the Rpg 1specific probe RSB228 confirmed these results (Fig. 2). The hybridizing band was the same size (approximately $4.5 \mathrm{kbp}$ ) as observed in cultivar Morex and the wild barley accession OSU6 (2) containing an intact Rpgl gene. These data were not in agreement with the stem rust phenotypes as only Hv672 was resistant to both pathotypes MCCF and HKHJ. OSU6 was previously characterized as resistant to stem rust pathotype MCCF, but multiple subsequent assays with both pathotypes MCCF and HKHJ on the selected plants used in the molecular analysis showed that

TABLE 2. Primers used to amplify Morex-like and Sm89010-like gene fragments for genotyping and sequencing

\begin{tabular}{|c|c|c|c|c|}
\hline \multirow[b]{2}{*}{ Fragment name $^{\mathrm{a}}$} & \multirow[b]{2}{*}{ Primer name } & \multirow[b]{2}{*}{ Primer sequence } & \multicolumn{2}{|c|}{ Location and product size ${ }^{b}$} \\
\hline & & & Sm89010 & Morex \\
\hline $1 \mathrm{Sm}$ & $37 F 2 / 228 R 6$ & cttccactttcctctctctcagatc/cggaaggcgctcaaagcagtctc & $-52-1,626 / 1,678 \mathrm{bp}$ & None \\
\hline 1 & 228F11/228R11 & tgcagcagtactctgcccacttgag/gggatgggtggctttgttgacaac & None & $-135-736 / 870$ bp \\
\hline 3 & 228F1/228R1 & gccggggctggacgatgaggaattc/gaactcgaatgcaaactccettgtc & $1,420-2,506 / 1,085 \mathrm{bp}$ & $884-1,967 / 1,082 \mathrm{bp}$ \\
\hline 4 & $228 \mathrm{~F} 2 / 228 \mathrm{R} 2$ & tgtgtagacattccaccegaagag/catctgtcccaatcaagtttgcagga & $2,151-3,247 / 1,095 \mathrm{bp}$ & $1,612-2,708 / 1,095 \mathrm{bp}$ \\
\hline 5 & $228 \mathrm{~F} 3 / 228 \mathrm{R} 3$ & catgcaacgaggaagccttgacgac/acccgatctactggtgatctct & $2,893-3,980 / 1,086 \mathrm{bp}$ & $2,353-3,440 / 1,086 \mathrm{bp}$ \\
\hline RT-PCR2 & $37-F 2 / 228 \mathrm{R} 7$ & cttccactttcctctctctcagatc/ttactgatgtaaagaccaccgtata & $52-2,065 / 879 \mathrm{bp}$ & None \\
\hline Seq $1 \mathrm{Sm}$ & DGK-F1 & cctcggatgctatcctcagcatgac & 875 & $\mathrm{NA}^{\mathrm{c}}$ \\
\hline Seq $1 \mathrm{Sm}$ & DGSex2 & tggggtaatgagaaaggtgtgatag & 953 & $\mathrm{NA}^{\mathrm{c}}$ \\
\hline Ubiquitin & Hvu_Ubi1_cw1/Hvu_Ubi1_ccw1 & gccgcaccetcgccgactac/cggcgttggggcactcettc & $219 \mathrm{bp}$ & $219 \mathrm{bp}$ \\
\hline
\end{tabular}

a Fragments 1 to 7 primers were used to amplify the Morex Rpgl coding regions as shown in Figure 1. Fragment 1 Sm primers amplified fragment 1 only from the Sm89010-like Rpg1 allele. Fragment 2 primers amplified fragment 2 from both Morex and Sm type Rpg1 alleles, but a higher molecular weight fragment from the Sm89010-like allele. Reverse transcription-polymerase chain reaction (RT-PCR) 1 and 2 primers were used to amplify a specific Rpg1 mRNA fragment from either Morex or Sm89010, respectively. Seq1Sm primers DGK-F1 and DGSex2 are internal to the location of primers 37F2 and 228R6, and were used to cover the sequencing gap in fragment $1 \mathrm{Sm}$, which was approximately $1.7 \mathrm{kbp}$.

${ }^{b}$ Bases upstream of the start codon are designated with minus (-) values. The product size is given in base pairs and the location indicated by base pair position in Morex or Sm89010 with the first base in the ATG start codon designated as 1. Ubiquitin primers were used to amplify a 219 bp fragment of the ubiquitin 1 gene from all cDNAs as shown in Figure 4.

${ }^{c}$ NA, not applicable.

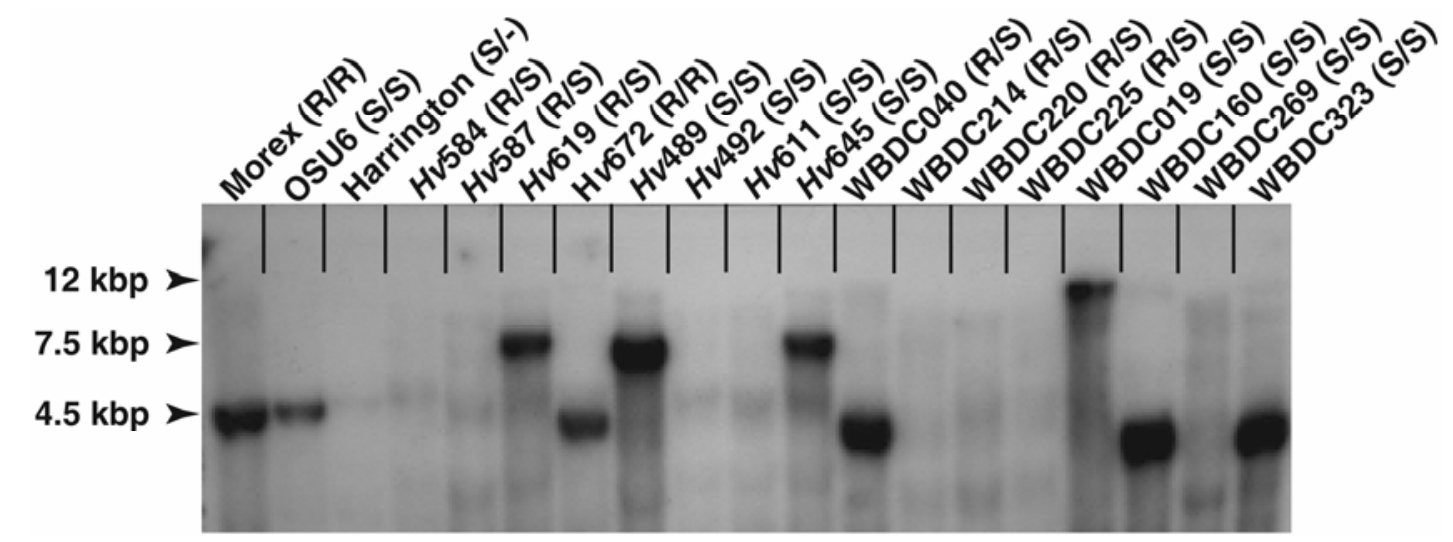

Fig. 2. Southern blot analysis of Hordeum accessions. Genomic DNA of each line was digested with HindIII and hybridized with the Rpg1-specific probe RSB228. The Morex-specific Rpgl band is approximately $4.5 \mathrm{kbp}$. The Sm89010-specific Rpgl band is approximately $7.5 \mathrm{kbp}$ (not shown) and of similar size to fragments hybridizing with DNA of Swiss landraces $H v 619, H v 489$, and $H v 645$. Harrington does not contain Rpgl. The R or S designation within parentheses indicate resistance or susceptibility to Puccinia graminis f. sp. tritici pathotypes MCCF/HKHJ, respectively. 
it is susceptible (B. Steffenson, unpublished data). Accession WBDC040 was resistant to moderately resistant to pathotype MCCF and susceptible to pathotype HKHJ, while WBDC323 was susceptible to both pathotypes.

All of the PCR-amplified genomic fragments were sequenced and aligned to resolve the phenotype versus genotype discrepancy. The predicted amino acid sequence of the resistant Swiss landrace Hv672 was identical to that of cultivar Morex RPG1, supporting the phenotype results to both stem rust pathotypes.

Rpg1 sequence analysis of wild barley accession WBDC040 suggested the presence of an inactive Rpgl gene since it had a GTT nucleotide insertion at nucleotide position 1,546 relative to the Morex sequence, a motif indicative of a susceptible allele (5). The GTT insertion resulted in an $\mathrm{S}$ to $\mathrm{R}$ amino acid substitution at position 319 and an F insertion at 320 (Fig. 3). Initial RT-PCR analysis suggested that WBDC040 produced an Rpgl mRNA similar to Morex (Fig. 4). However, sequencing of several cDNA clones of the ninth exon/intron junction of Rpgl from Morex and the resistant wild barley accession WBDC040 showed that there was a substitution altering the conserved $3^{\prime}$ AG splice site of intron 9 to $\mathrm{AA}$ at position 3251 resulting in the next downstream AG being used as the $3^{\prime}$ splice site. This resulted in a 17-nucleotide deletion in the mRNA of the WBDC040 allele, causing a frame shift and a stop codon at amino acid position 617 (Fig. 5). Therefore, the resistance of WBDC040 to pathotype MCCF could not be due to the Rpgl gene. This was confirmed by protein analyses that showed that the RPG1 protein failed to accumulate in WBDC040 and autophosphorylation was not detected (Fig. 6). These results suggest the presence of a gene different from Rpgl conferring resistance to pathotype $\mathrm{MCCF}$ in this accession. The susceptibility of WBDC040 to pathotype HKHJ supports this result.

Rpgl sequence analysis of wild barley accession WBDC323 identified a few nucleotide substitutions scattered throughout the nucleotide sequence in addition to the GTT insertion as previously described. At the amino acid level, however, there are only two differences in the Rpgl sequence between WBDC323 and Morex. The GTT insertion results in an $\mathrm{S}$ to $\mathrm{R}$ substitution at position 319, an $\mathrm{F}$ insertion at position 320 , and an $\mathrm{I}$ to $\mathrm{F}$

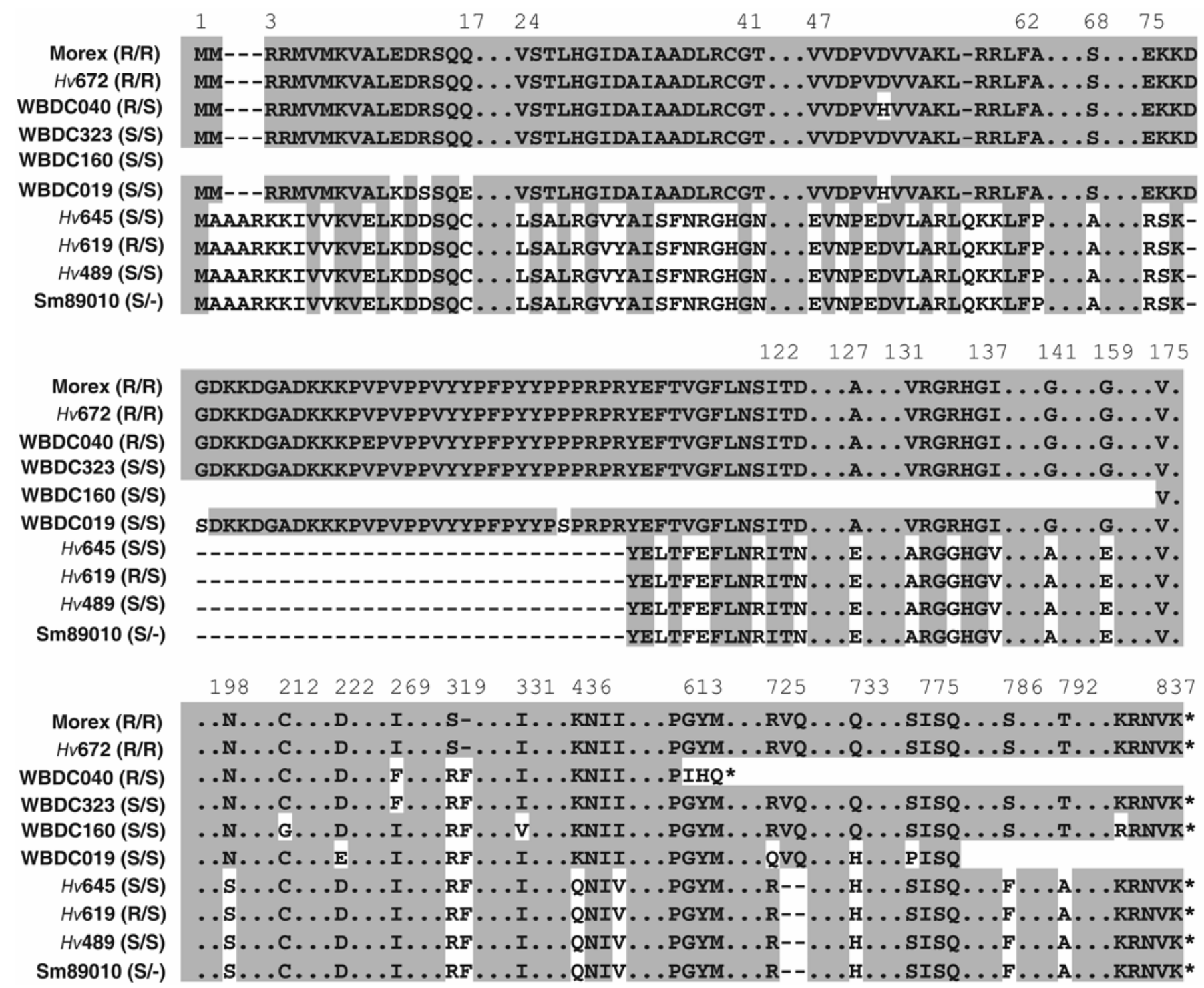

Fig. 3. Predicted amino acid alignment of Rpg1 alleles from Swiss barley landraces and wild barley accessions compared with Morex and Sm89010. Gray boxes represent identical residues and white areas represent diverged residues compared to the resistant Morex Rpg1 allele. Dashes represent gaps in the alignment. Dots represent blocks of conserved amino acid sequences between all alleles. Asterisk indicates stop codon. The R or S designation within parentheses indicate resistance or susceptibility to Puccinia graminis f. sp. tritici pathotypes MCCF/HKHJ, respectively. Sequence information has been deposited in the GenBank database under the following accession numbers: Swiss Hv489, DQ854803; Swiss Hv619, DQ854802; Swiss Hv645, DQ854804; Swiss Hv672, DQ657356; WBDC019, DQ854805; WBDC040, DQ657357; WBDC160, DQ854806; and WBDC323, DQ657358. The Morex and Sm89010 sequences have been previously deposited and can be found under accession numbers AF509748 and AF509762, respectively (2). 
substitution at position 269 (Fig. 3). RT-PCR analysis showed that the Rpgl gene was expressed in this accession at the mRNA level (Fig. 4), and western blot and autophosphorylation assays showed that the RPG1 protein is present and capable of autophosphorylation (Fig. 6). Though the GTT insertion and/or the I to F substitution did not affect the autophosphorylation activity, the protein was not functional in conferring resistance to either pathotype MCCF or HKHJ.

Lines with an apparent unequal recombination. We previously described an apparent unequal recombination event in

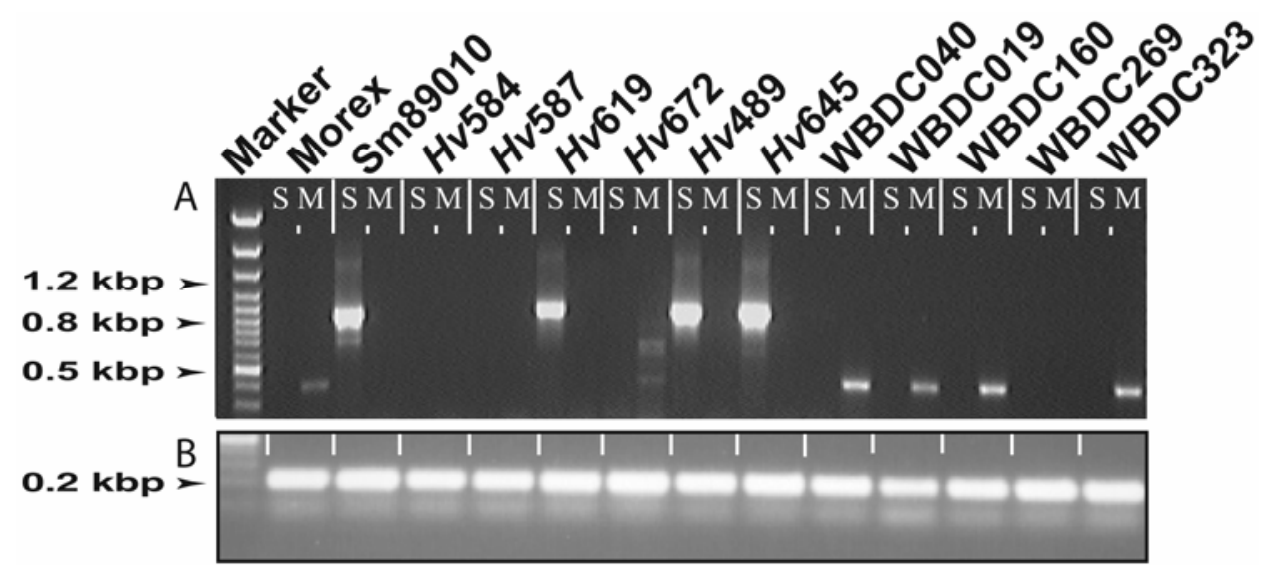

Fig. 4. Reverse transcription-polymerase chain reaction showing Morex-like or Sm89010-like bands. A, Oligo dT-primed cDNA derived from total RNA was amplified with either Morex-like Rpg1-specific primers (right lane designated M) or Sm89010-like Rpg1-specific primers (left lane designated S). The accessions used for cDNA isolation are listed above. The Morex band is approximately $0.4 \mathrm{kbp}$ and the Sm89010 band is approximately $0.9 \mathrm{kbp}$. Lines devoid of Rpg 1 did not yield a band with either set of primers. B, Oligo dT-primed cDNA of each was amplified using ubiquitin-specific primers to demonstrate loading and RNA quality.

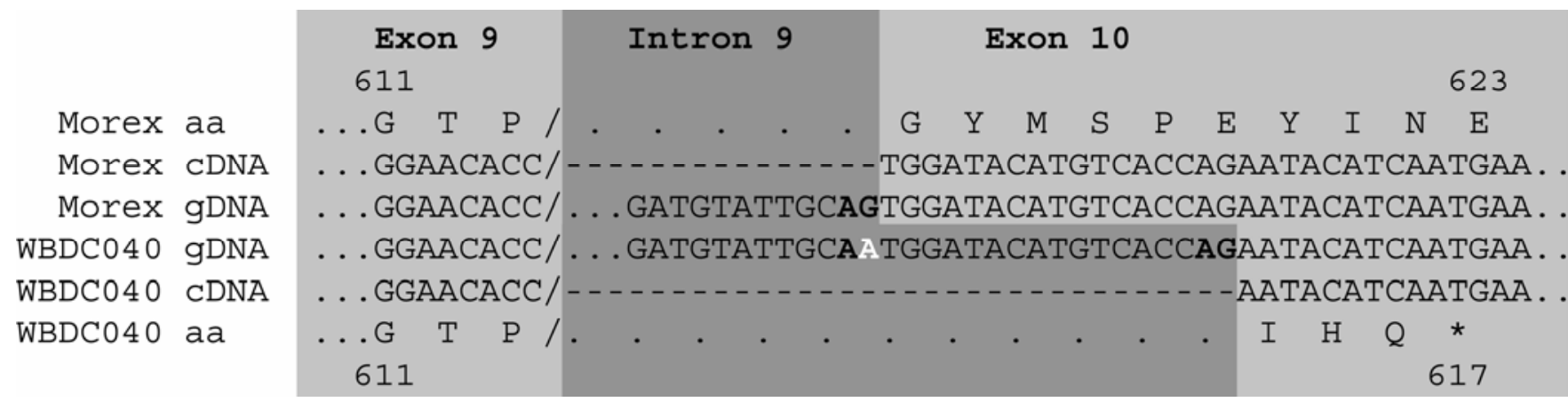

Fig. 5. Sequence comparison of Morex and WBDC040 Rpg1 intron 9/exon 10 junction and predicted amino acid sequence. The WBDC040 cDNA sequence is based on three cloned reverse transcription-polymerase chain reaction generated fragments. Note the G to A substitution at the $3^{\prime}$ end of intron 9 (white A) in the WBDC040 genomic sequence and the subsequent use of the next AG pair (AG bold) for splicing of the mRNA. The resulting cDNA is missing 17 nucleotides from exon 10 causing a frame shift and a stop codon at position 617. The truncated translation product failed to accumulate a protein detectable by the RPG1specific antibody.

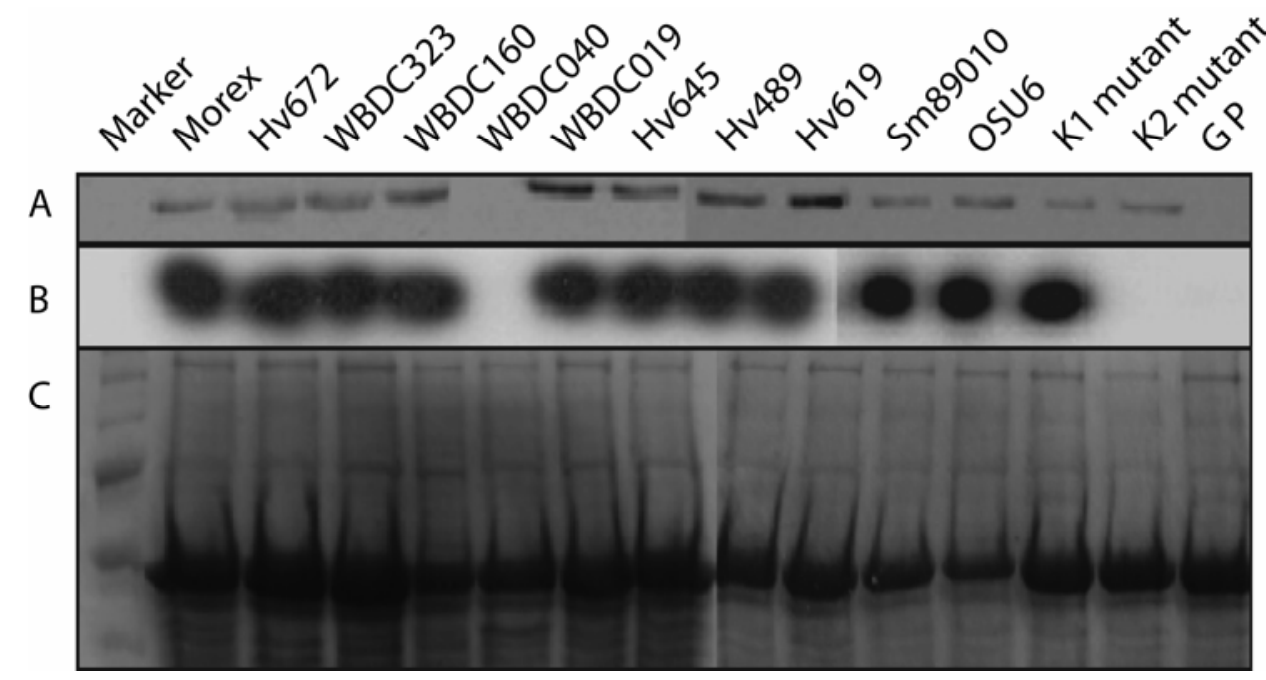

Fig. 6. Western blot and autophosphorylation assays. A, RPG1 protein visualized with an RPG1-specific antibody. WBDC040 and GP (cultivar Golden Promise, which does not have the Rpg1 gene) failed to produce a detectable RPG1 protein. B, Autophosphorylation assay corresponding to RPG1 western blot. WBDC040 and GP did not autophosphorylate. The K2 (kinase domain 2) mutant also failed to autophosphorylate, while the K1 (kinase domain 1) mutant autophosphorylated as previously reported (13). C, Coomassie blue staining of total protein to demonstrate protein loading in each lane. 
barley line Sm89010, resulting in a chimeric gene between ABC1037, a closely linked Rpgl homologue, and Rpg1 (1). In the Swiss landraces Hv619, Hv489, and Hv645, amplification of fragments 3 to 7 with the Morex-specific primers was successful and of the expected size, but fragment 1 failed to amplify (Fig. 1). Fragment 2 amplified, but was larger than from Morex (1.6 kbp versus $1.0 \mathrm{kbp}$ ). This was similar to the situation previously observed with the Sm89010 allele. Successful amplification of fragment 1 using Sm89010 recombinant allele-specific primers confirmed the presence of an Sm89010-like recombinant allele in these lines (2). Southern blot hybridization of HindIII digested Hv619, Hv489, and Hv645 DNA yielded a band similar to that previously observed with Sm89010 (1) and with a higher molecular weight than from the lines containing the Morex-like allele (Fig. 2). RT-PCR amplification using primers specific to Morex or Sm89010 alleles resulted only in a band similar to Sm89010 in these lines and no amplification with Morex-specific primers (Fig. 4). Nucleic acid sequence in silico translation predicted amino acid identity among the three Swiss landraces Hv619, Hv489, and Hv645 and with the Sm89010 Rpgl-like recombinant gene (Fig. 3). At the nucleotide level, there were only three differences between the entire Sm89010 Rpgl gene sequence and the sequences of the three Swiss landraces (2). There was a $\mathrm{G}$ to A substitution at position 2,183 as well as single nucleotide deletions of $\mathrm{A}$ at 2,199 and $\mathrm{C}$ at 4,713, with numbering based on the Sm89010 Rpgl sequence (data not shown). These single nucleotide polymorphisms (SNPs) did not change the amino acid sequence since the $\mathrm{G} 2,183$ A substitution was in the third nucleotide in the codon and A 2,199 and C 4,713 deletions were in introns. The recombinant Rpgl-like gene is not expected to provide stem rust resistance as previously demonstrated with the Sm89010 line (1). Landraces Hv489 and Hv645 were indeed susceptible to stem rust pathotypes MCCF and HKHJ as expected; however, Hv619 was resistant to pathotype MCCF, suggesting the presence of a gene different from Rpgl conferring the resistance. The lack of a functional Rpgl gene in Hv619 also was confirmed by susceptibility to pathotype HKHJ. All three lines with the recombinant type Rpgl allele produced an RPG1 antibody positive protein and autophosphorylated (Fig. 6).

Lines with apparent $3^{\prime}$ and $5^{\prime}$ deletions in the Rpg1 genes. The wild barley accessions WBDC019 and WBDC160 were susceptible to stem rust pathotypes MCCF and HKHJ and had apparent $3^{\prime}$ and 5' deletions in the Rpg1-like genes. For WBDC019, fragment 7 (the most $3^{\prime}$ Rpgl PCR fragment) failed to amplify and for WBDC160, fragments 1 and 2 (the most 5' Rpgl PCR fragments) failed to amplify with either the Morex- or Sm89010specific primers (Fig. 1B). Southern blot analysis showed that both lines had Rpgl probe-positive hybridizing fragments; however, accession WBDC019 showed a very high molecular weight fragment, while the WBDC160 fragment was about the same size as Morex (Fig. 2). Since WBDC019, the only accession lacking amplification for fragment 7 , had a unique high molecular weight fragment hybridized, we assumed that there might be a large insertion in this region. Additional efforts using amplification conditions for long range PCR $(\approx 10 \mathrm{kbp}$ fragments $)$ and fragment 7 primers (228F12/228R12) did not result in amplification. Long range PCR also was applied for amplification of fragment 1 and 2 from WBDC160 using primers 228F11/228R11 and 228F6/228R6, respectively, but without success. RT-PCR produced a band similar to Morex (Fig. 4), indicating that the gene is expressed and suggesting the possibility of an mRNA similar to the Morex-like allele. The RT-PCR was carried out with primers that amplified the internal Morex-like region of the gene. Therefore, the bands are approximately the same size as the Morex Rpgl mRNA.

The amplified genomic DNA fragments 1 to 6 from WBDC019 and fragments 3 to 7 from WBDC160 were sequenced and aligned with the other RPG1 derived amino acid sequences (Fig.
3). The susceptible Rpg1 allele from WBDC019 had diverged, including the GTT insertion, but still was very similar to the Morex RPG1 protein sequence until the $\mathrm{C}$ terminus where it could no longer be amplified and recognized as a Morex Rpgl homologue. All attempts at extending the sequence with $3^{\prime}$ RACE failed.

The in silico translated sequence of the susceptible wild barley WBDC160 RPG1 starts at amino acid position 162 and is fairly similar to the Morex sequence from then on (Fig. 3). This gene also has the GTT insertion resulting in substitution of S 319 R and insertion of F 320. Both lines produced RPG1 antibodypositive protein and autophosphorylated (Fig. 6).

Rpg1 gene PCR amplification deficient lines. Of the 16 lines analyzed, 8 failed to produce any amplification products with either Morex-specific or Sm89010-specific Rpgl primers (Fig. 1B). The eight lines were evenly divided between the germplasm groups with four Swiss landraces and four wild barley accessions failing to yield any amplification products. To confirm the absence of Rpgl alleles in the eight genotypes, an additional 27 combinations of primers designed from the Morex and Sm89010 nucleotide sequences were tested on this group. No amplification products were obtained with these sets of primers, confirming the absence of a Morex-like or Sm89010-like Rpg1 in these lines (data not shown). In accordance with the PCR amplification results, no hybridization to the RSB228 probe was observed with DNA from lines lacking PCR amplification (Fig. 2). To further confirm the lack of an Rpgl-like gene, two Swiss landraces resistant (Hv584 and Hv587) and one wild barley accession susceptible (WBDC269) to pathotype MCCF from this group were included in RT-PCR analysis. No amplification products were obtained using Morex or Sm89010 Rpg1 primers (Fig. 4). Five of these accessions (Hv584, Hv587, WBDC214, WBDC220, and WBDC225) were resistant to pathotype MCCF and three (Hv492, Hv611, and WBDC269) were susceptible. All of these accessions were susceptible to pathotype HKHJ. These results clearly indicate the presence of a stem rust resistance gene(s) different from Rpgl in these Hordeum accessions.

\section{DISCUSSION}

To investigate the diversity and possible origin of the barley stem rust resistance gene $\operatorname{Rpg} 1$, we analyzed two subsets of $H$. vulgare and $H$. vulgare subsp. spontaneum germplasm: one comprised of eight Swiss landraces and the second of eight wild barley accessions from diverse geographic regions. Accessions resistant and susceptible to stem rust pathotype MCCF were chosen in order to identify functional Rpgl variants as well as spontaneous mutations that result in loss of function (Table 1). Additionally, pathotype HKHJ also was used in this study because it is highly effective for detecting Rpgl in the presence of other stem rust resistance genes.

The Swiss landraces used in this study were a potential source of stem rust resistant germplasm and possibly new Rpgl alleles. However, only one accession (Hv672) with an intact Rpgl gene was found to confer resistance to both $P$. graminis f. sp. tritici pathotypes MCCF and HKHJ (Fig. 1; Table 1). The gene in Hv672 was identical in predicted amino acid sequence to the cultivar Morex RPG1 protein (Fig. 3). Thus, Swiss landraces appear to be the sole source for Rpgl as both cultivars Chevron and Peatland from Vorrenwald Eich in Canton Lucerne (18) and accession Hv672 from Surava in Canton Graubünden (B. Steffenson, unpublished data) have intact Rpgl genes that encode a protein with amino acid sequences identical to Morex, and are all functional in conferring resistance to pathotype HKHJ. It is interesting to note that Hv672 (together with its duplicate selection Hv671) was the only accession among 74 Swiss landraces tested that exhibited resistance to pathotype HKHJ (B. Steffenson, unpublished data). Thus, the presence of Rpgl also is 
apparently rare in Swiss landraces. The Rpgl-derived amino acid sequence in Kindred was previously shown to be identical to that of Chevron and Peatland (2). As mentioned previously, the donor of Rpgl in Kindred was likely Chevron.

Wild barley is known to be a rich source of different disease resistance alleles for cultivated barley improvement (8). However, to stem rust, the frequency of resistance is relatively low, even in a large (318 accessions) and diverse collection (20). To identify the possible source of Rpgl in cultivated barley, eight ecogeographically diverse wild barley accessions that were resistant or susceptible to stem rust pathotype MCCF were selected for this study. Analysis of the Rpgl alleles from the eight wild barley accessions failed to identify any with an intact and functional Rpg1 gene as was found for the Swiss landrace accession Hv672 (Fig. 1 and Table 1). Surprisingly, most of the resistant wild barley accessions contained either a defective Rpgl gene or did not contain a recognizable $R p g l$ gene. This result indicates that other stem rust resistance gene(s) effective against $P$. graminis f. sp. tritici pathotype MCCF exist in wild barley. The fact that defective Rpgl alleles were found among the wild barley accessions indicates that the gene exists or has existed in these populations.

A curious GTT indel occurs in the Rpgl gene of several Swiss landraces and wild barley accessions. This indel results in an $S$ $319 \mathrm{R}$ substitution and insertion of $\mathrm{F}$ at position 320 (Fig. 3). In the wild barley accession WBDC323, the only other amino acid change relative to the Morex RPG1 sequence is an I $269 \mathrm{~F}$ substitution resulting in a stem rust susceptible phenotype. We have previously observed the GTT indel in the wild barley accession OSU6 (PBI004-7-0-015 accession no. 8321) (2). OSU6 was previously characterized as resistant to stem rust pathotype MCCF, but multiple subsequent assays with both pathotypes MCCF and HKHJ on the selected plants used in the molecular analysis showed that it is susceptible (B. Steffenson, unpublished data). Except for the amino acid changes resulting from the GTT indel, OSU6 does not have any other differences in the RPG1 amino acid sequence compared to the Morex RPG1 sequence. The $\mathrm{S}$ to RF change due to the GTT indel appears in seven out of the eight accessions containing an Rpgl-like gene analyzed here and thus is very common (Fig. 3). These seven accessions (Hv619, Hv489, Hv645, WBDC040, WBDC019, WBDC160, and WBDC323) do not show a consistent pattern of resistance or susceptibility to the stem rust pathotype MCCF, but all were susceptible to HKHJ, the best pathotype for indicating the functionality of Rpgl. Thus, it seems that the GTT insertion affects Rpgl-mediated stem rust resistance as indicated by reaction to pathotype HKHJ, but may not affect resistance conferred by other genes, i.e., those conferring resistance to pathotype MCCF. The potential role of the GTT insertion is unknown. It occurs at position 319 in the RPG1 amino acid sequence, placing it near the end of the kinase 1 domain. This domain is not essential for RPG1 autophosphorylation activity, but plays an important, yet undefined role in the overall stem rust resistance reaction (13). The fact that the RPG1 protein with the GTT insertion is active in autophosphorylation suggests that the kinase 2 domain, which is essential for autophosphorylation and disease resistance, is intact and the gene is expressed and may retain some unknown function (Fig. 6).

A set of primers that can differentiate barleys with and without Rpgl was developed based on the gene's nucleotide sequence and the GTT insertion (5). The authors tested 42 different barley cultivars and showed that the primers correctly identified the phenotype in all cases, but one. The one exception was cultivar Maud, originally classified as resistant (with a question mark), but grouped with susceptible lines that do not contain an Rpgl gene (5). We repeatedly tested Maud at both the seedling and adult plant stages and found it to be susceptible to stem rust pathotype MCCF (B. Steffenson, unpublished data). These results support our conclusion that the GTT indel is associated with a nonfunc- tional Rpgl gene, at least in terms of P. graminis f. sp. tritici resistance to pathotypes MCCF and HKHJ.

Another interesting group of Rpgl alleles are those represented by an apparent unequal recombination between the Rpgl gene and a closely linked paralog designated ABC1037 (1). This recombination was observed only in the Swiss landraces and not in any of the wild barley lines tested. The gene is expressed at the mRNA level (Fig. 4). Moreover, an RPG1 antibody positive protein is produced and is active in autophosphorylation (Fig. 6). The function, if any, of this recombinant allele is unknown, but we speculate that it may involve recognition of a putative AvrRpgl effector of the pathogen. Recombination among homologous disease resistance genes has been observed and can result in the creation of novel resistance phenotypes $(14,17)$.

The two wild barley accessions WBDC019 and WBDC160 with apparent $3^{\prime}$ and $5^{\prime}$, respectively, incomplete $R p g 1$ genes were susceptible to both stem rust pathotypes as expected (Fig. 1 and Table 1). Both genes, however, are expressed at the mRNA level (Fig. 4) and produce an RPG1 antibody positive protein that undergoes autophosphorylation (Fig. 6). The genomic nucleotide sequence of the Rpgl gene in these lines is apparently similar enough to the Morex Rpgl gene in the interior regions to produce mRNA and protein with Rpgl properties and an intact kinase domain 2 that is active in autophosphorylation. The failure to amplify Rpgl fragment 7 from WBDC019 and fragments 1 and 2 from WBDC160 suggest that those regions of the Rpg1 gene may be deleted, harbor a large insertion, or are highly diverged. A 19 nucleotide insertion and 11 nucleotide deletion in intron 12, plus a 17 nucleotide deletion at the $3^{\prime}$ end and 25 nucleotide deletion at the $5^{\prime}$ end of exon 13 of the WBDC019 allele, indicated high divergence in this region of the gene. However, these changes apparently are far enough downstream of the kinase 2 domain and do not affect the protein's autophosphorylation activity (Fig. 6). The reason for the extensive divergence of the sequence at the $3^{\prime}$ end while the rest of the Rpgl gene primary structure is mostly conserved is unknown.

The origin of the widely used and durable Rpgl gene bred into North American barley cultivars remains a mystery since we have yet to discover a wild barley accession containing an intact and functional Rpg1 gene. Although the number of wild barley accessions examined by complete sequencing to date is small, they nevertheless represent some of the most stem rust resistant germplasm found to date in extensive evaluations of $H$. vulgare sp. spontaneum germplasm $(8,20)$. In wild barley, we repeatedly found an Rpgl gene with a very similar primary gene structure, but containing a GTT insertion that apparently renders the gene nonfunctional in providing stem rust resistance. Is this the progenitor resistance gene that later developed specificity for the stem rust pathotypes MCCF/HKHJ by deletion of the GTT triplet in the cultivated species? A similar, but inversed situation has been described with the Pto disease resistance gene (10). In that case, the wild tomato relative $L$. pimpinellifolium contributed the functional resistance gene, but the domesticated $L$. esculentum has maintained highly related and expressed pto and fen alleles. These alleles encode predicted proteins of 87 and $98 \%$ identity with the PTO and FEN kinases and are active in in vitro autophosphorylation, but not functional in disease resistance. The answer to the question of the Rpgl origin will require a more extensive search of wild barley germplasm for a potential Rpg1 donor.

Eight of the 16 Hordeum accessions analyzed in this study did not have an Rpg1-like gene recognizable by multiple PCR primers or Southern blot analysis, yet five exhibited some resistance to pathotype MCCF (Fig. 1 and Table 1). These results clearly indicate the presence of a gene(s) different from Rpgl conferring resistance to stem rust pathotype MCCF. The observed resistance could be due to the rpg4/Rpg 5 complex as there is some evidence that one or both of these genes confers resistance to pathotype 
MCCF in addition to other pathotypes (21)(B. Steffenson, unpublished data). Additional studies are underway to elucidate the genetics of stem rust resistance in these accessions.

In summary, Rpgl alleles with a primary structure different from the one found in cultivar Morex, but apparently functional in conferring stem rust resistance to pathotypes MCCF and HKHJ were not discovered in this study. Moreover, we failed to find a progenitor Rpgl gene among the wild barley accessions examined. A common potential progenitor gene has a GTT indel that apparently results in failure to provide resistance to pathotype MCCF. Further, examples of an apparent unequal recombination were discovered, but only in the domesticated Hordeum group (i.e., Swiss landraces) suggesting that this may be a relatively recent event. We have previously shown that RPG1 autophosphorylation is essential, but alone is not sufficient for conferring stem rust resistance (13). The observation that all the lines examined in this study, except WBDC040, were active in autophosphorylation reinforces the previous data that it is not sufficient for resistance. Thus, RPG1 plays a complex role in the stem rust disease resistance-signaling pathway.

\section{ACKNOWLEDGMENTS}

We thank G. M. Anderson and S. Dahl for technical assistance and J. Valkoun (International Center for Agricultural Research in the Dry Areas, Aleppo Syria) and G. Kleijer (Service de Genetique Swiss Federal Research Station for Plant Production, Nyon, Switzerland) for the gift of the wild barley and landrace barley germplasm, respectively. Research was supported by USDA-NRI grant No. 2004 35301-14635, the United States Barley Genome Project, and Lieberman-Okinow Endowment at the University of Minnesota.

\section{LITERATURE CITED}

1. Brueggeman, R., Drader, T., and Kleinhofs, A. 2006. The barley serine/threonine kinase gene Rpgl providing resistance to stem rust belongs to a gene family with five other members encoding kinase domains. Theor. Appl. Genet. 113:1147-1158.

2. Brueggeman, R., Rostoks, N., Kudrna, D., Kilian, A., Han, F., Chen, J., Druka, A., Steffenson, B., and Kleinhofs, A. 2002. The barley stem rustresistance gene Rpgl is a novel disease-resistance gene with homology to receptor kinases. Proc. Natl. Acad. Sci. USA 99:9328-9333.

3. Buckler, E. S., IV, and Thornsberry, J. M. 2002. Plant molecular diversity and applications to genomics. Curr. Opin. Plant Biol. 5:107-111.

4. Chomczynski, P., and Sacchi, N. 1987. Single-step method for RNA isolation by acid guanidium thiocyanate-phenol-chloroform extraction. Anal. Biochem. 162:156.

5. Eckstein, P., Rossnagel, B. G., and Scoles, G. 2003. Allele-specific markers within the barley stem rust resistance gene (Rpg1). Barley Genet. Newsl. 33:7-11.
6. Edwards, K., Johnstone, C., and Thompson, C. 1991. A simple and rapid method for the preparation of plant genomic DNA and PCR analysis. Nucleic Acids Res. 19:1351.

7. Ellis, J. G., Lawrence, G. J., Luck, J. E., and Dodds, P. N. 1999. Identification of regions in alleles of the flax rust resistance gene $\mathrm{L}$ that determine differences in gene-for-gene specificity. Plant Cell 11:495-506.

8. Fetch, T. G., Steffenson, B. J., and Nevo, E. 2003. Diversity and sources of multiple disease resistance in Hordeum spontaneum. Plant Dis. 87:1439-1448.

9. Horvath, H., Rostoks, N., Brueggeman, R., Steffenson, B., von Wettstein, D., and Kleinhofs, A. 2003. Genetically engineered stem rust resistance in barley using the Rpg1 gene. Proc. Natl. Acad. Sci. USA 100:364-369.

10. Jia, Y., Loh, Y.-T., Zhou, J., and Martin, G. B. 1997. Alleles of Pto and Fen occur in bacterial speck-susceptible and fenthion-insensitive tomato cultivars and encode active protein kinases. Plant Cell 9:61-73.

11. Kleinhofs, A., Kilian, A., Saghai Maroof, M. A., Biyashev, R. M., Hayes, P., Chen, F. Q., Lapitan, N., Fenwick, A., Blake, T. K., Kanazin, V., Ananiev, E., Dahleen, L., Kudrna, D., Bollinger, J., Knapp, S. J., Liu, B., Sorrells, M., Heun, M., Franckowiak, J. D., Hoffman, D., Skadsen, R., and Steffenson, B. J. 1993. A molecular, isozyme and morphological map of the barley (Hordeum vulgare) genome. Theor. Appl. Genet. 86:705712 .

12. Kolmer, J. A. 2001. Early research on the genetics of Puccinia graminis and stem rust resistance in wheat in Canada and the United States. Pages 51-82 in: Stem Rust of Wheat: From Ancient Enemy to Modern Foe. P. D. Peterson, ed. American Phytopathological Society, St. Paul, MN

13. Nirmala, J., Brueggeman, R., Maier, C., Clay, C., Rostoks, N., Kannangara, C. G., von Wettstein, D., Steffenson, B., and Kleinhofs, A. 2006. Subcellular localization and functions of the barley stem rust resistance receptor-like serine/threonine-specific protein kinase Rpg1. Proc. Natl. Acad. Sci. USA 103:7518-7523.

14. Richter, T. E., Pryor, T. J., Bennetzen, J. L., and Hulbert, S. H. 1995. New rust resistance specificities associated with recombination in the $R p 1$ complex in maize. Genetics 141:373-381.

15. Richter, T. E., and Ronald, P. C. 2000. The evolution of disease resistance genes. Plant Mol. Biol. 42:195-204.

16. Riely, B. K., and Martin, G. B. 2001. Ancient origin of pathogen recognition specificity conferred by the tomato disease resistance gene Pto. Proc. Natl. Acad. Sci. USA 98:2059-2064.

17. Song, W.-Y., Pi, L.-Y., Wang, G.-L., Gardner, J., Holsten, T., and Ronald, P. C. 1997. Evolution of the rice Xa21 disease resistance gene family. Plant Cell 9:1279-1287.

18. Steffenson, B. J. 1992. Analysis of durable resistance to stem rust in barley. Euphytica 63:153-167.

19. Steffenson, B. J., Miller, J. D., and Yin, Y. 1993. Detection of the stem rust resistance gene Rpgl in barley seedlings. Plant Dis. 77:626-629.

20. Steffenson, B. J., Olivera, P., Roy, J. A., Jin, Y., Smith, K. P., and Muehlbauer, G. J. 2007. A walk on the wild side: Mining wild wheat and barley collections for rust resistance genes. Aust. J. Agric. Res. 58:532544.

21. Sun, Y., and Steffenson, B. J. 2005. Reaction of barley seedlings with different stem rust resistance genes to Puccinia graminis f. sp. tritici and P. graminis f. sp. secalis. Can. J. Plant Pathol. 27:80-89.

22. Williams, K. J. 2003. The molecular genetics of disease resistance in barley. Aust. J. Agric. Res. 54:1065-1079. 